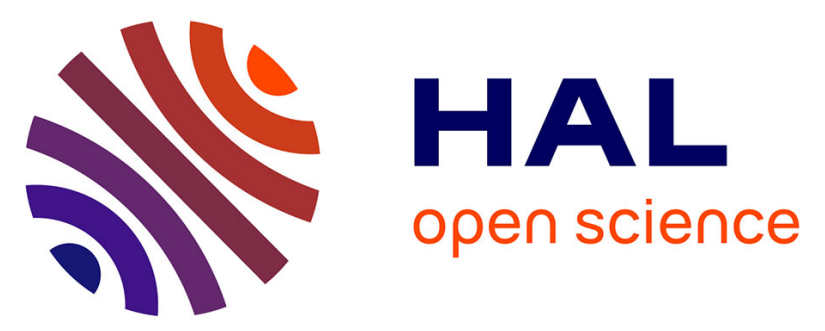

\title{
Pathological findings and prostate specific antigen outcomes after radical prostatectomy in men eligible for active surveillance-does the risk of misclassification vary according to biopsy criteria?
}

Guillaume Ploussard, Laurent J. Salomon, Evanguelos Xylinas, Yves Allory, Dimitri Vordos, Andras Hoznek, Claude-Clément Abbou, Alexandre de La Taille

\section{- To cite this version:}

Guillaume Ploussard, Laurent J. Salomon, Evanguelos Xylinas, Yves Allory, Dimitri Vordos, et al.. Pathological findings and prostate specific antigen outcomes after radical prostatectomy in men eligible for active surveillance-does the risk of misclassification vary according to biopsy criteria?. Journal of Urology, 2010, 183 (2), pp.539-44. 10.1016/j.juro.2009.10.009 . inserm-00453763

\section{HAL Id: inserm-00453763 https://www.hal.inserm.fr/inserm-00453763}

Submitted on 5 Feb 2010

HAL is a multi-disciplinary open access archive for the deposit and dissemination of scientific research documents, whether they are published or not. The documents may come from teaching and research institutions in France or abroad, or from public or private research centers.
L'archive ouverte pluridisciplinaire HAL, est destinée au dépôt et à la diffusion de documents scientifiques de niveau recherche, publiés ou non, émanant des établissements d'enseignement et de recherche français ou étrangers, des laboratoires publics ou privés. 
Pathological findings and PSA outcomes after radical prostatectomy in men eligible for active surveillance: does the risk of misclassification vary according to the different biopsy criteria?

Guillaume Ploussard, Laurent Salomon, Evanguelos Xylinas, Yves Allory, Dimitri Vordos, Andras Hoznek, Claude-Clément Abbou, Alexandre de la Taille

INSERM U955 eq07 Departments of Urology and Pathology, APHP, CHU Henri Mondor, Créteil, France

Correspondence:

Dr A. de la Taille

INSERM U955 Eq07 Department of Urology, CHU Mondor

51 avenue du Maréchal de Lattre de Tassigny, 94000 Créteil, France

Tel: 33149812254 / Fax: 33149812568

Email: adelataille@hotmail.com

No financial disclosure.

Keywords: prostate cancer; radical prostatectomy; active surveillance; low risk

Word count abstract:

Word count text : 


\begin{abstract}
Purpose:

To evaluate and compare the pathological findings and the PSA outcomes after radical prostatectomy (RP) in men eligible for AS, according to 3 different biopsy inclusion criteria.

Materials and Methods:

The study population included 177 men eligible for AS who fulfilled clinico-biological criteria (Gleason score $\leq 6, \mathrm{PSA}<10$, clinical stage T1c) and biopsy criteria as follows: $(1)<3$ positive cores and $<3 \mathrm{~mm}$ of total tumour length; (2) and/or $<3$ positive cores with a cancer involvement $<50 \%$ in any core; (3) and/or $<33 \%$ of positive cores. PSA density cut-offs of 15 and $20 \mathrm{ng} / \mathrm{ml} / \mathrm{gr}$ were also studied among these groups. Pathological findings on RP specimens and biochemical recurrence-free survival (RFS) were studied. Median follow-up was 34 months.

Results:

Cancers were graded Gleason 7 on RP specimens from $48.3 \%$ to $55.4 \%$ of cases. The rates of extracapsular extension (ECE) and vesicle seminal invasion (SVI) ranged from $11.2 \%$ to $17.5 \%$, and from $1.1 \%$ to $1.8 \%$, respectively, regardless of PSA density. The use of PSA density as AS criterion decreased for AS by 1.4-fold and by 2.3-fold the number of men eligible according to the cut-off used. The risk of unfavourable disease (defined as pT3-4 stage and/or a Gleason score $\geq 8$ ) remained between $15 \%$ to $19.2 \%$ when a PSA density cut-off of $15 \mathrm{ng} / \mathrm{ml} / \mathrm{gr}$ was used. The risk of overall unfavourable disease was significantly higher in men with a cancer involvement $\geq 3 \mathrm{~mm}$ in initial biopsy compared with men who fulfilled these most stringent biopsy criteria $(27.3 \%$, vs $13.5 \%$, respectively; $p=0.023$ ). The 3 -year biochemical RFS was $91.5 \%$ and was not affected by the 3 different AS definitions.

Conclusions:

Even with the use of a 21-core biopsy protocol, the rate of unfavourable disease on RP specimens remains still elevated in men eligible for AS. Patients must be informed of this risk of misclassification which is about $20 \%$ in men who fulfil the less stringent biopsy criteria.
\end{abstract}




\section{INTRODUCTION}

The prostate cancer $(\mathrm{PCa})$ detection rate has strongly increased since the PSA screening and the use of extended prostate biopsy protocol. Active surveillance entails a strategy by which selected men are managed expectantly with the intention to apply potentially curative treatment in case of progression signs [4]. Cancers that are amenable to AS usually are identified on favourable preoperative parameters and the risk is estimated by integrating Gleason score, pretreatment PSA, clinical stage, prostate volume and the extent of biopsy involvement with tumour [6-10]. Published AS series use different criteria largely based on centre experiences and preferences with no hard data. The most common clinical data used to define AS criteria are a Gleason score $\leq 6, \mathrm{PSA} \leq 10 \mathrm{ng} / \mathrm{ml}$ and a clinical stage T1c disease. The PSA density is noted as inclusion criteria in some studies with different reported cut-offs for AS inclusion [6,13-14, Carter]. Other characteristics to consider include pathological biopsy parameters with a wide variation concerning the AS inclusion criteria. A cancer involvement of $<33 \%$ of biopsy cores was retained in the UCSF cohort [16]. Other series included cancers involving $<3$ cores only [14] and with an extent of cancer in any core $<50 \%[13,17]$. Recently, a prospective trial of AS (SURACaP trial) has been opened in France including patients with a tumour length $<3 \mathrm{~mm}$ in $<3$ cores [18]. Studies comparing entry criteria for AS protocols, especially in terms of biopsy parameters, are needed to clarify the best candidates for active surveillance. Recent studies emphasized the risk of under-diagnosis, adverse pathological findings and thus, missing window of curability if AS is preferred [Suardi, Conti]. Based on these limitations and the wide variation of existing pathological criteria for AS inclusion, we decided to compare pathological findings and PSA outcome after radical prostatectomy (RP) in men eligible for AS, according to 3 different biopsy parameters reported in the literature. PSA density cut-offs which have been published in recent AS studies were also tested among each group in analyses. 
Between January 2001 and July 2008, we identified patients who were diagnosed with PCa in a set of 21 biopsies and who have undergone a RP for clinically localized and low-risk prostate cancer according to preoperative data. Inclusion criteria for low-risk cancers were PSA level $\leq 10 \mathrm{ng} / \mathrm{ml}$, a clinical stage T1c disease, a Gleason score $\leq 6$ and a life-expectancy $>10$ years. All patients underwent clinical evaluations, including digital rectal examinations, serum PSA, and transrectal ultrasound. All the patients had undergone a 21-core biopsy protocol as previously described for abnormal digital rectal findings or elevated PSA [19]. All the biopsies and radical prostatectomies were performed in our department and specimens were evaluated by senior uropathologists. Tumour volume was not measured routinely. Data from clinical evaluation, biopsy and RP specimens, and follow-up were recorded in a prospective database. PSA recurrence was defined as PSA $>0.2 \mathrm{ng} / \mathrm{ml}$ after $\mathrm{RP}$.

Of all the patients in the database, we identified 177 patients who met the main favourable criteria (PSA $\leq 10 \mathrm{ng} / \mathrm{ml}$, T1c disease, Gleason score $\leq 6$, life-expectancy $>10$ years) and who were diagnosed to have a cancer involvement of $<33 \%$ of biopsy cores (group 3 ). Of these 177 men, 112 met the following criteria: $<3$ positive cores with a cancer involvement $<50 \%$ in any core (group 2). Of these 112 men, 89 met the following criteria: $<3$ positive cores with total tumour length $<3 \mathrm{~mm}$ (group 1).

We studied the pathological findings on RP specimens, such as Gleason score, extracapsular extension (ECE), seminal vesicle invasion (SVI), positive surgical margins, and the PSA outcomes in the 3 groups during the follow-up. Statistical analyses were conducted between the 3 groups regardless of PSA density, and then, with the use of 2 PSA density cut-offs : 15 and 20 $\mathrm{ng} / \mathrm{ml} / \mathrm{gr}$. Secondly, we compared the results between men of group 1 (cancer involvement $<3$ $\mathrm{mm}$ ) and men who did not fulfil this most stringent biopsy criteria (not group 1). The qualitative data were tested using a chi-square test or Fisher's exact test as appropriate and the quantitative date were tested using Student's t-test. The Mann-Whitney's test was used in case of no normal distribution. Biochemical recurrence-free survival was established using the Kaplan-Meier method. Curves were tested by log-rank test. The limit of statistical significance was defined as $\mathrm{p}<0.05$. The SPSS 13.0 (Chicago, Illinois) software was used for analysis. 


\section{RESULTS}

Of the 468 patients with PCa in a 21 -core biopsy scheme and who have undergone a RP, 177 , 112 and 89 men fulfilled preoperatively the AS criteria of group 3,2 and 1, respectively.

Table 1 shows the patients' characteristics. The 3 groups were comparable and there were no significant baseline differences between the 3 groups in terms of PSA level, age, DRE findings, prostate volume and PSA density.

Table 2 summarizes the pathological findings at RP and the rates of unfavourable disease according to each AS criteria. The use of PSA density as AS criterion decreased by 1.4 -fold and by 2.3-fold the number of men eligible for AS, if a cut-off of 20 and $15 \mathrm{ng} / \mathrm{ml} / \mathrm{gr}$ was used, respectively. A majority of Gleason score 6 was observed in group $1(51.7 \%)$, whereas a majority of Gleason score 7 or more was reported in groups $2(53.6 \%)$ and $3(55.4 \%)$. The rates of unfavourable pathological findings increased in groups 2 and 3 compared with the rates in group 1. Primary Gleason score 4 was reported in $12.4 \%$ of men of group 3 (versus $8.9 \%$ in group 1). Extracapsular extension was noted in $17.5 \%$ of RP specimens in group 3, versus $11.2 \%$ in group 1. The rate of positive surgical margins reached $20.9 \%$ in group 3 compared with $18.0 \%$ in group 1. Globally, when unfavourable was defined as ECE, SVI and/or Gleason score 8 or more, the overall rate was $20.3 \%$ in group 3 and $13.5 \%$ in group 1. T3 or T4 cancers were reported in $12.4 \%$ of cases in group 1 and $19.2 \%$ of cases in group 3 . No differences reached significance when comparing these rates between groups.

Table 3 lists pathological features when PSA density was also used as AS criterion (cut-offs of 20 and $15 \mathrm{ng} / \mathrm{ml} / \mathrm{gr}$ ). Risks of unfavourable disease were then slightly lower but differences did not reach significance.

Table 4 shows the statistical comparisons when analysis compared men who fulfilled the group 1 criteria with the remaining men (not group 1). Significant differences were noted. Men who did not fulfilled the group 1 criteria had statistically more frequently PCa with ECE ( $p=0.025), p T 3-4$ stage $(p=0.020)$ and unfavourable disease $(p=0.023)$. The use of a PSA density cut-off of 20 did not modify these statistical differences. Statistical significance was not found when the cut-off was decreased to $15 \mathrm{ng} / \mathrm{ml} / \mathrm{gr}$. These men had also more high-grade PCa and positive surgical margins, but difference did not reach significance.

No differences appeared between the groups in terms of seminal vesicle invasion and PSA failure after RP.

The overall 3-year recurrence-free survival rate was $91.5 \%$. All the biochemical recurrences occurred during the 2 first years after RP. Figure 1 shows the biochemical recurrence-free survival curves according to the 3 groups. The survival rates between the men in group $1(n=89)$ and the men who did not fulfilled the group 1 criteria $(n=88)$ were not statistically different (logrank test, $p=0.571$, see Figure 2 ). The 3 -year recurrence-free survival rate was $92.7 \%$ for the men in group 1 and $89.8 \%$ for men who did not fulfilled the group 1 criteria. 


\section{DISCUSSION}

Active surveillance is a treatment option for selected patients with low-risk PCa. Data demonstrate that the proportion of low-risk men electing surveillance has risen in recent years [20]. Published AS series used different inclusion criteria largely based on centre experiences and preferences with no hard data. A wide variation concerning the biopsy inclusion criteria is also expressed in the literature. Many AS criteria have been based on series of patients diagnosed with much less than 21-core biopsies, sometimes even sextant biopsies only. The number of positive cores, the tumour length (total or at any core) and the percent of cancer involvement at any core are predictive factors of tumour volume in RP specimens or biochemical failure after RP [8,22-23].

The aim of our retrospective study was to compare the rate of misclassification associated with the use of 3 different biopsy criteria [13,16-17]. For each criterion, we also tested the impact of PSA density on the number of men eligible and the risk of unfavourable disease [van den Bergh, Carter]. A non organ-confined disease and/or a high-grade PCa defined a misclassification case. The 3 groups were comparable in terms of biopsy Gleason score (no grade 4 or 5), PSA level, age and DRE findings. Each patient underwent the same 21-core biopsy protocol under local anaesthesia. Thus, the different studied groups were strongly homogeneous.

The overall results of our series indicate that a cancer of low grade and small volume in biopsies is not necessarily indicative of a good pathological assessment on RP specimens. The Gleason score was upgraded from $48.3 \%$ to $55.4 \%$ according to the different biopsy criteria. The rate of misclassification, ie the presence of either non organ-confined disease or high grade PCa was relatively high. Unfavourable disease defined as ECE, SVI or primary Gleason 4 was reported $27.7 \%$ of cases in group 3 . The less stringent the biopsy criteria were, the higher the misclassification rates were. When unfavourable disease was defined as ECE, SVI and/or Gleason score 8 or more, this risk in group 3 was increased by 1.5 -fold compared with the risk of group 1. Of the 97 men who fulfilled the group 2 and 3 criteria but not the stringent group 1 criteria (total tumour length $>3 \mathrm{~mm}$ ), about one third had unfavourable disease on RP specimens. This rate was only $13.5 \%$ when group 1 criteria were considered. Men who did not fulfil the group 1 criteria had statistically more frequently PCa with ECE, pT3-4 stage and unfavourable disease. However, concerning the biochemical recurrence after RP, the estimate risk of relapse was comparable and not significantly different in the 3 groups (about $5 \%$ ).

Using the risk-stratification schemes based only on PSA level, DRE findings, biopsy Gleason score and extent of cancer involvement appears insufficient to identify cancers with a low risk of progression. Therefore, other factors, such as PSA density and PSA velocity, would provide additional significance $[6,8]$. In our cohort, patients who had a high PSA density at diagnosis were more likely to have unfavourable disease on RP specimens. Thus, the difference was slight and the use of PSA density as AS criterion did not strongly modify our results. Only $41 \%$ patients in our series had a PSA density lower than $0.15 \mathrm{ng} / \mathrm{ml} / \mathrm{gr}$ and only 64 men fulfilled at once this measurement and the group 1 criteria. Ideally, the strictest criteria should be used in order to decrease the risk of unfavourable disease. However, very high selective and stringent AS inclusion criteria allows the inclusion of a relatively small proportion of patients from the pool of those with localized PCa. Avoiding misclassification errors need to reduce severely the number of men eligible for AS. In our series, when the rate of misclassification was reduced from $27.7 \%$ to $21.3 \%$ (ie, by 1.3 -fold), the number of men eligible for AS decreased by 2 -fold (from 177 to 89 men).

Rates of misclassification and non-organ confined disease were lower than those reported in recent studies [Conti, Suardi]. These discrepancies can be explained by non comparable cohorts, retrospective analyses or other biases. However, we think that the use of a 21-core biopsy scheme, compared with a 10-12 cores protocol, has a strong impact on this improvement. The counterpart is that the saturation biopsy strategies have been hypothesized to increase the potential risk of overtreating patients whose tumours induce very low risk to life. Nevertheless, the role of prostatic biopsies changed. The actual importance of prostate biopsies has evolved from purely cancer detection to investigating as to how biopsy results can assist clinical management 
for patients. The inclusion of patients in AS protocols emphasizes the necessity of perfectly accurate staging strategies.

Biochemical recurrence rates were probably not a valid endpoint to address conclusions in men eligible for AS. The PSA failures can not reflect accurate measurement in men who did not undergo radical treatment. However, PSA failure is established to be associated with an increased risk of progression to metastatic disease and specific death [Freedland]. Few PSA failures occurred in our series and the biochemical recurrence-free survival was $91.5 \%$ at 3 years after surgery. None of the patients developed clinical recurrence with symptoms during the followup and no specific death appeared. These results may validate the use of AS as treatment option in selected patients who are informed of the need of close surveillance and the risk of deferred treatment. However, the number of events in all 3 groups was small and conclusions on differences in biochemical RFS may therefore be too premature. The lack of significant differences might be due to short follow-up since a large proportion of PSA failures occur beyond 3-year follow-up [Amling].

Our cohort can appear small, compared with the study of Suardi et al. [11]. However, Suardi et al. have considered only PSA, Gleason score and DRE as AS criteria. We added pathological indicators of cancer involvement (percent of cancer in any core, total tumor length) and the impact of PSA density in a very homogeneous and extensively biopsied cohort. One of the other limitations of our study was to consider for the PSA analyses only the men who have undergone RP. This may represent a bias, the target population for AS being larger than our restricted cohort. In this study, we tested current AS criteria which were internationally used or recently published [Carter, 13,16-18]. Our listing of AS criteria was not complete but provided a representative cohort of the men actually included in AS protocols. Recent AS criteria of the French protocol were interesting as it provided the most stringent definition in terms of biopsy parameters [18]. However, only prospective studies comparing biopsy inclusion criteria for AS protocols and comparing AS with immediate radical treatment would be able to clarify the best candidates for active surveillance according outcomes in terms of rising PSA and specific deaths.

Even with the use of a 21-core extended biopsy scheme, a number of PCa will be selected for AS, while these actually constitute more aggressive tumors. However, these results do not contraindicate the inclusions in AS protocol. AS protocols always included a close surveillance scheme, aiming to catch the aggressive tumors as soon as possible during follow-up. Nevertheless, our findings can help urologists to better inform the patients about the risk of misclassification and the need of close surveillance. 


\section{CONCLUSIONS}

Our study demonstrates the limitations of AS inclusion criteria, even with the use of a 21-core biopsy protocol. The rate of unfavourable disease remains still elevated, even with the use of the most selective criteria. In the group of patients with the most stringent biopsy criteria, one fifth of patients have non organ-confined disease and/or primary Gleason score of grade $4 / 5$. Thus, any AS selective model provides potential limitations with a risk of under-diagnosis, adverse pathological findings and potential hazards of missing window of curability if AS is preferred. These results can help urologists to better inform the men eligible for AS about this risk of misclassification. 


\section{REFERENCES}

1. Etzioni R, Penson DF, Legler JM, di Tommaso D, Boer R, Gann PH, et al. Overdiagnosis due to prostate-specific antigen screening: lessons from U.S. prostate cancer incidence trends. J Natl Cancer Inst 2002;94(13):981-90

2. Stamey TA, Freiha FS, McNeal JE, Redwine EA, Whittemore AS, Schmid HP. Localized prostate cancer. Relationship of tumor volume to clinical significance for treatment of prostate cancer. Cancer 1993;71(3):933-8

3. Johansson JE, Holmberg L, Johansson S, Bergström R, Adami HO. Fifteen-year survival in prostate cancer. A prospective, population-based study in Sweden. JAMA 1997;277(6):467-71

4. Klotz L. Active surveillance for prostate cancer: for whom? J Clin Oncol 2005;23(32):8165-9

5. Bill-Axelson A, Holmberg L, Filén F, Ruutu M, Garmo H, Busch C, et al. Scandinavian Prostate Cancer Group Study Number 4. Radical prostatectomy versus watchful waiting in localized prostate cancer: the Scandinavian prostate cancer group-4 randomized trial. J Natl Cancer Inst 2008;100(16):1144-54

6. Epstein JI, Walsh PC, Carmichael M, Brendler CB. Pathologic and clinical findings to predict tumor extent of nonpalpable (stage T1c) prostate cancer. JAMA 1994;271(5):368-74

7. Kattan MW, Eastham JA, Wheeler TM, Maru N, Scardino PT, Erbersdobler A, et al. Counseling men with prostate cancer: a nomogram for predicting the presence of small, moderately differentiated, confined tumors. J Urol 2003;170(5):1792-7

8. Nakanishi H, Wang X, Ochiai A, Trpkov K, Yilmaz A, Donnelly JB, et al. A nomogram for predicting low-volume/low-grade prostate cancer: a tool in selecting patients for active surveillance. Cancer 2007;110(11):2441-7

9. Steyerberg EW, Roobol MJ, Kattan MW, van der Kwast TH, de Koning HJ, Schröder FH. Prediction of indolent prostate cancer: validation and updating of a prognostic nomogram. J Urol 2007;177(1):107-12

10. Ochiai A, Trpkov K, Yilmaz A, Donnelly B, Babaian RJ. Validation of a prediction model for low volume/low grade cancer: application in selecting patients for active surveillance. J Urol 2007;177(3):907-10

11. Suardi N, Capitanio U, Chun FK, Graefen M, Perrotte P, Schlomm T, et al. Currently used criteria for active surveillance in men with low-risk prostate cancer: an analysis of pathologic features. Cancer 2008 Oct;113(8):2068-72

12. Chun FK, Haese A, Ahyai SA, Walz J, Suardi N, Capitanio U, et al. Critical assessment of tools to predict clinically insignificant prostate cancer at radical prostatectomy in contemporary men. Cancer 2008 Aug;113(4):701-9

13. van As NJ, Norman AR, Thomas K, Khoo VS, Thompson A, Huddart RA, et al. Predicting the probability of deferred radical treatment for localised prostate cancer managed by active surveillance. Eur Urol 2008;54(6):1297-305

14. Roemeling S, Roobol MJ, Postma R, Gosselaar C, van der Kwast TH, Bangma CH et al. Management and survival of screen-detected prostate cancer patients who might have been suitable for active surveillance. Eur Urol 2006;50(3):475-82 
15. van den Bergh RC, Roemeling S, Roobol MJ, Aus G, Hugosson J, Rannikko AS, et al. Outcomes of Men with Screen-Detected Prostate Cancer Eligible for Active Surveillance Who Were Managed Expectantly. Eur Urol 2008 [Epub ahead of print]

16. Dall'Era MA, Konety BR, Cowan JE, Shinohara K, Stauf F, Cooperberg MR, et al. Active surveillance for the management of prostate cancer in a contemporary cohort. Cancer 2008;112(12):2664-70

17. Bastian PJ, Mangold LA, Epstein JI, Partin AW. Characteristics of insignificant clinical T1C prostate tumors. A contemporary analysis. Cancer 2004;101(9):2001-5

18. Mottet N, Irani J. Active surveillance in prostate cancer: the French protocol. Prog Urol 2008 18(4 Suppl FMC):F12-16

19. de la Taille A, Antiphon P, Salomon L, Cherfan M, Porcher R, Hoznek A, et al.

Prospective evaluation of a 21-sample needle biopsy procedure designed to improve the prostate cancer detection rate. Urology 2003;61(6):1181-6

20. Cooperberg MR, Broering JM, Kantoff PW, Carroll PR. Contemporary trends in low risk prostate cancer: risk assessment and treatment. J Urol 2007;178(3):14-9

21. Cheng L, Poulos CK, Pan CX, Jones TD, Daggy JK, Eble JN, et al. Preoperative prediction of small volume cancer (less than $0.5 \mathrm{ml}$ ) in radical prostatectomy specimens. J Urol 2005;174(3):898-902

22. Greene KL, Elkin EP, Karapetian A, Duchane J, Carroll PR, Kane CJ; CaPSURE Investigators. Prostate biopsy tumor extent but not location predicts recurrence after radical prostatectomy: results from CaPSURE. J Urol 2006;175(1):125-9

23. Ochiai A, Troncoso P, Chen ME, Lloreta J, Babaian RJ. The relationship between tumor volume and the number of positive cores in men undergoing multisite extended biopsy:

implication for expectant management. J Urol 2005;174(6):2164-8 


\section{LEGENDS}

Table 1. Patients characteristics at baseline $(n=194)$

Table 2. Pathological findings and PSA outcomes after radical prostatectomy according to the 3 different biopsy criteria (groups 1,2 and 3).

Table 3. Univariate analysis comparing pathological findings and PSA outcomes between group 1 $(n=94)$ and group 3 excluding group $1(n=94)$.

Figure 1. Biochemical recurrence-free survivals according the 3 different biopsy criteria (groups 1,2 and 3). 